\title{
Peripheral Arterial Desaturation Is Further Exacerbated by Exercise in Adolescents With Acute Mountain Sickness
}

\author{
Sarah A. Major, DPhil; Ryan J. K. Hogan, MBChB; Elizabeth Yeates, BM; Chris H. E. Imray, FRCS \\ From University Hospital Southampton National Health Service Foundation Trust, Southampton, United Kingdom (Dr Major); Brighton and \\ Sussex University Hospitals National Health Service Trust, Brighton, United Kingdom (Dr Hogan); Otago District Health Board, Dunedin, \\ New Zealand (Dr Yeates); and Warwick Medical School, University Hospitals Coventry and Warwickshire National Health Service Trust, \\ Coventry, United Kingdom (Dr Imray).
}

Objective.-Rapid ascent to altitude can result in the development of high altitude illnesses such as acute mountain sickness (AMS). This study aimed to investigate AMS symptoms in adolescents and study basic cardiopulmonary measurements at altitude.

Methods.-Thirty-eight adolescents aged 16 to 19 years flew to $3500 \mathrm{~m}$ from $215 \mathrm{~m}$ and continued over a 23-day period to ascend to a maximum altitude of $5200 \mathrm{~m}$. Each member of the expedition completed a Lake Louise Self-Assessment Questionnaire (LLSAQ) on a daily basis, and AMS was defined as a score of $\geq 3$, with an associated headache. Physiology measurements included a step test, and both before and after exercise pulse oximetry, blood pressure, and pulse rate.

Results.-Oxygen saturation inversely correlated with altitude $(P=.001)$. Mean pulse rate increased from 70 beats/min $( \pm 6.5)$ at $215 \mathrm{~m}$ to 83 beats/min $( \pm 2.2)$ at $3500 \mathrm{~m}(P=.01)$, and a rise in blood pressure with ascent was highlighted $(P=.004)$. The majority of subjects $(84 \%)$ had an LLSAQ of 3 or more on at least 1 occasion, and they tended to record higher pulse rates $(P=.005)$ and lower oxygen saturations $(P=.001)$. Exercise-induced drops in oxygen saturation and raised pulse rates were more prolonged in subjects with severe AMS compared with subjects not having AMS $(P=.046$ and $P=$ .005 , respectively).

Conclusions.-The LLSAQ scoring system appeared to be a simple and effective technique to aid the diagnosis of adolescents who have AMS, and it may help improve the safety of large groups traveling to altitude. The AMS subjects tended to have low oxygen saturations and high pulse rates, highlighting potential areas for further research.

Key words: acute mountain sickness, altitude, adolescents

\section{Introduction}

As foreign travel to remote locations becomes more popular, an increasing number of people, including adolescents, are ascending to high altitude environments. Altitude exposure can lead to a number of altituderelated illnesses, some of which are self-limiting whereas others are more serious. Numbers of persons affected are rising as travel to high altitude is increasing. The rate of ascent can affect the incidence of such conditions, and a wider recognition of the risks is to be encouraged.

Corresponding author: Sarah A. Major, DPhil, Southampton General Hospital, Tremona Road, Southampton S016 6YD, United Kingdom (e-mail: majorsarah@gmail.com).
On ascent to altitude, the atmospheric pressure falls. Although the fraction of oxygen in inhaled air $\left(\mathrm{FiO}_{2}\right)$ remains constant at $21 \%$, the oxygen partial pressure drops from approximately $160 \mathrm{~mm} \mathrm{Hg}$ at sea level to 103 $\mathrm{mm} \mathrm{Hg}$ at $3500 \mathrm{~m}$ : a reduction in available oxygen of $35 \%{ }^{1}$ Altitude illness can vary from simple headache that resolves spontaneously to high altitude pulmonary edema and high altitude cerebral edema, which require prompt treatment. Acute mountain sickness (AMS) is usually a self-limiting condition that develops shortly after ascent to high altitude and usually resolves after a few days of acclimatization. ${ }^{2}$ It can occur above approximately $2500 \mathrm{~m}$ and has the symptoms of headache, nausea, vomiting, fatigue, and sleep disturbances. Symp- 
toms are more likely to occur or become more severe at higher altitude and when ascent is rapid. ${ }^{3}$ Although acclimatization occurs if ascent to altitude is more gradual, some persons are inherently more susceptible to AMS.

Published studies of AMS show a wide range of incidences, among both adults and adolescents. A suggestion that adolescents are at greater risk of AMS than adults is supported by the results of some studies that found higher incidence rates among adolescent populations., ${ }^{4,5}$ A $27.0 \%$ incidence rate of AMS among adults at $4400 \mathrm{~m}$ was identified in 1 study, with the rate among adolescents in the group reported as $50.0 \% .^{4}$ Another study of adults and adolescents showed similar incidences: $25.0 \%$ and $45.0 \%$, respectively, on ascent to altitudes of $2000 \mathrm{~m}$ to $3000 \mathrm{~m} .{ }^{6}$ Even higher rates of AMS among adolescents have been shown by other studies, one of which identified a prevalence of $91.7 \%$ in a group of $15-$ to 18 -year-olds traveling to $5500 \mathrm{~m} .{ }^{5}$ The evidence is not conclusive, however, and many studies do not show this higher incidence among younger people. ${ }^{7,8}$ As recent studies show, the daily incidence even within a single study can vary markedly. Adolescents traveling to altitudes as high as $4100 \mathrm{~m}$ showed incidences of AMS ranging between $3.8 \%$ and $42.3 \% .^{9}$ The varying incidences reported in the literature highlight the challenge of understanding the mechanisms involved. The altitude reached, ascent rates, physical activity while at altitude, and factors such as fitness, comorbidities, and general health may all play a part.

The Lake Louise Self-Assessment Questionnaire (LLSAQ) was originally a research tool, ${ }^{10,11}$ but has been found to be potentially beneficial to trekking groups as a means of identifying persons displaying symptoms of AMS and of adapting the itinerary accordingly. The LLSAQ scores symptoms of headache, nausea, vomiting, fatigue, and sleep disturbances according to severity, and total scores for an individual subject are collated. The system has been used in many studies of adults at altitude, but fewer studies have investigated adolescents. ${ }^{5,9}$

The aim of this study was to record the symptoms of AMS in an adolescent population ascending to altitude. We further sought to investigate whether AMS, as diagnosed using the LLSAQ, was associated with particular trends in oxygen saturation, pulse rate, and blood pressure both before and after exercise.

\section{Methods}

The study was performed on a British Schools Exploring Society (BSES) group of young people traveling to altitude for the first time. All participants were healthy, taking no prophylactic drug treatments for AMS, and gave written informed consent to take part in the study. Written approval for the study was obtained from the Coventry and Warwickshire Research and Ethics Committee.

The participants traveled from the United Kingdom to New Delhi (at 215 m), where initial "sea level" measurements were taken as a control, and subsequently flew to Leh, Ladakh (at $3450 \mathrm{~m}$ ). Measurements were taken daily while the subjects were acclimatizing for 4 days in Leh and during subsequent ascent along the Thansglasgo and surrounding valleys. Thirty-eight adolescents took part and underwent an identical ascent profile for the initial 7 days of the expedition. One person left the expedition on day 7 owing to development of suspected pulmonary edema. The expedition then split into 2 groups. One group ( 9 females, 10 males) proceeded up a valley to a maximum height of approximately $5800 \mathrm{~m}$ and completed recordings to $5200 \mathrm{~m}$ over an additional

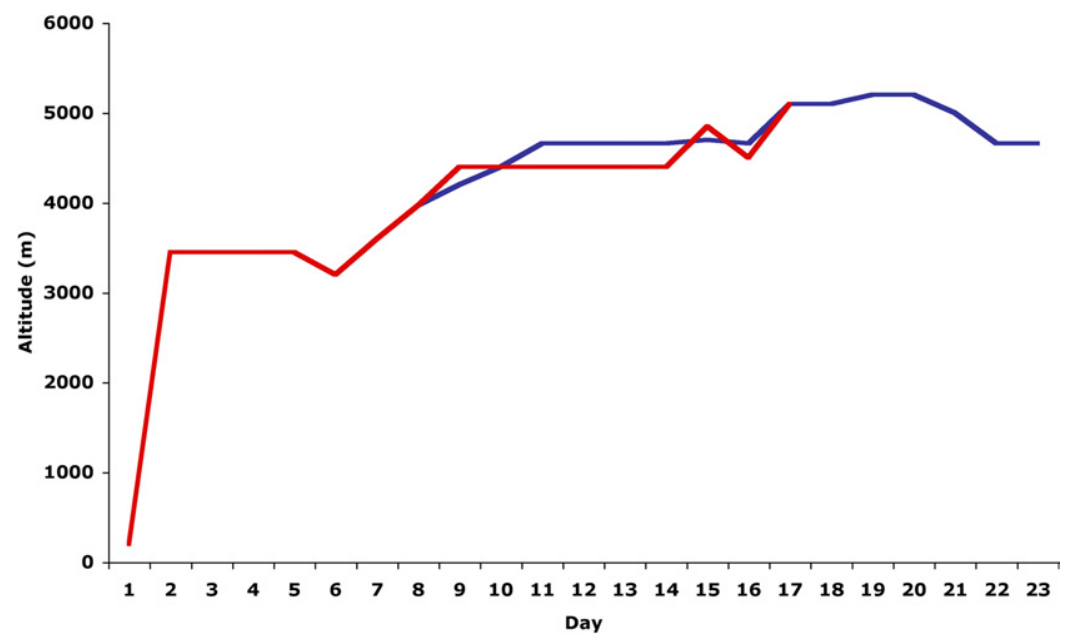

Figure 1. Expedition altitude ascent profiles. Group 1 (blue line) recorded data for 23 days, and group 2 (red line) recorded data for 17 days. 
16 days. The second group (4 females, 14 males) ascended an adjacent valley to a maximum height of approximately $6000 \mathrm{~m}$ and continued measurements for a further 9 days up to $4700 \mathrm{~m}$. The ascent profile for the first 23 days is outlined in Figure 1.

\section{SELF-ASSESSMENT OF SYMPTOMS}

A questionnaire based on the LLSAQ scoring system ${ }^{10,11}$ was designed. The LLSAQ investigated 5 different symptoms: headache, dizziness/light headedness, gastrointestinal symptoms, fatigue, and sleep disturbance. Each symptom was scored from 0 to 3 depending on severity. Each participant was provided with a booklet containing the questionnaire and asked to fill out the grid with relevant symptoms on a daily basis. That was performed every morning of the expedition before breakfast. The AMS was defined as a total score of 3 or more after a recent gain in altitude, with an associated headache. These scores were further subdivided into mild AMS (a score of 3 to 4 with an associated headache), moderate AMS (a score of 5 to 6 with an associated headache), and severe AMS (a score of 7 or more with an associated headache). Participants recording a score of 3 or more on a particular day were "highlighted" to the trek leaders for more detailed assessment.

\section{OXYGEN SATURATION AND PULSE RATE}

Oxygen saturation and pulse rate measurements were taken using Nonin Onyx 9550s pulse oximeters (Nonin Medical Inc, North Plymouth, MN). Measurements were taken on right index fingers and performed outside the tents in the morning before breakfast. "At rest" readings were recorded after 5 minutes of inactivity while sitting down. A period of exercise was then undertaken. Participants stepped on and off a $25 \mathrm{~cm}$ high step, stepping in time with a metronome set at 60 beats per minute. The exercise was performed for 2 minutes, and oxygen saturation and pulse rate measurements were taken and recorded immediately thereafter. Further sets of measurements were taken after a 1-minute rest period. This protocol was similar to the Caudwell Xtreme Everest protocol. $^{12}$

\section{BLOOD PRESSURE}

Blood pressure measurements were taken on a daily basis before breakfast, after 5 minutes of inactivity. Post exercise measurements taken directly after stepping were not taken owing to technical constraints; measurements were recorded after 1 minute of rest. Omron 705IT oscillometric sphygmomanometers (Omron Corporation, Kyoto, Japan) were used for the study.

\section{STATISTICS}

All physiological results are expressed as mean $( \pm 95 \%$ $\mathrm{CI}$ ), and difference between means was performed with $t$ test analysis. The LLSAQ scores as ranked data are presented as median values, with score comparisons achieved using 2-tailed Mann-Whitney $U$ test. Spearman's rank correlation was used for associations of LLSAQ scores and physiological measurements (IBM SPSS statistics, version 19). Each daily recording of LLSAQ is coupled to the physiological measurements for that individual participant on that day, giving a pool of direct comparisons of symptoms to physiological signs. Statistical significance was judged as $P<.05$.

\section{Results}

The study was performed with 38 adolescents aged 16 to 19 years old, with a mean age of $17.4( \pm 0.34)$. Twentyfive participants were male, of mean age $17.5( \pm 0.44)$ years, and 13 were female, of mean age $17.2( \pm 0.5)$ years. All participants reside at a home elevation of $<500 \mathrm{~m}$.

Completion rates were $97.8 \%$ for the initial 16 days of the expedition. Thirty-seven adolescents completed the LLSAQ and physiological measurements for the first 16 days, with 1 person completing 7 days of measurements before being evacuated with suspected pulmonary edema. Measurements were missed on certain occasions across all altitude ranges owing to non-altitude-related illness or equipment availability, with measurement completion rates of $89.1 \%$. A group of 19 participants (9 female and 10 male) completed measurements for an additional 7 days, up to a maximum height of $5200 \mathrm{~m}$. The rest of the party stopped taking measurements after day 16 owing to prioritization of mountaineering objectives.

The overall AMS incidence for the group as determined by LLSAQ was $84.0 \%$, with 32 of 38 subjects recording scores $\geq 3$ at least once during the expedition. There were no significant differences in incidence between males and females: $80.1 \%$ and $92.3 \%$, respectively. Both groups had a median of 2 episodes per person $(P=.252$ Mann-Whitney $U$ test). On initial ascent to $3500 \mathrm{~m}$, the LLSAQ scores markedly increased in all 5 categories, with a median LLSAQ score of 2.0 (Table 1 and Figure 2). The highest incidence of AMS was recorded on day 2. An increase in incidence of AMS was observed at approximately $4660 \mathrm{~m}$ initially, but 
Table 1. Lake Louise Self-Assessment Questionnaire scores for the expedition

\begin{tabular}{|c|c|c|c|c|c|c|c|c|c|c|}
\hline Day & Altitude (m) & $n$ & $H$ & $D$ & $G I$ & $F$ & $S$ & Median LLSAQ & AMS recorded ( $n)$ & Daily incidence (\%) \\
\hline 1 & 215 & 38 & 0 & 0 & 0 & 0 & 0 & 0 & 0 & 0 \\
\hline 2 & 3450 & 38 & 25 & 28 & 13 & 31 & 16 & 2.0 & 15 & 39.0 \\
\hline 3 & 3450 & 38 & 19 & 6 & 3 & 11 & 18 & 1.5 & 10 & 26.0 \\
\hline 4 & 3450 & 38 & 15 & 7 & 7 & 15 & 23 & 1.0 & 5 & 13.0 \\
\hline 5 & 3450 & 38 & 17 & 4 & 5 & 5 & 12 & 1.0 & 4 & 10.5 \\
\hline 6 & 3200 & 38 & 6 & 6 & 9 & 6 & 9 & 0 & 4 & 10.5 \\
\hline 7 & 3600 & 38 & 9 & 5 & 7 & 10 & 9 & 0 & 3 & 7.9 \\
\hline 8 & 3970 & 37 & 6 & 7 & 5 & 9 & 8 & 1.0 & 2 & 5.4 \\
\hline 9 & 4200 & 37 & 10 & 4 & 5 & 14 & 15 & 1.0 & 4 & 10.8 \\
\hline 10 & 4400 & 37 & 11 & 6 & 7 & 9 & 12 & 1.0 & 6 & 16.2 \\
\hline 11 & 4660 & 37 & 13 & 12 & 9 & 12 & 14 & 1.0 & 10 & 27.0 \\
\hline 12 & 4660 & 37 & 9 & 3 & 4 & 11 & 10 & 1.0 & 6 & 16.2 \\
\hline 13 & 4660 & 37 & 8 & 4 & 3 & 8 & 11 & 0 & 4 & 10.8 \\
\hline 14 & 4660 & 37 & 5 & 6 & 2 & 7 & 9 & 0 & 3 & 8.1 \\
\hline 15 & 4700 & 37 & 6 & 5 & 1 & 9 & 11 & 0 & 2 & 5.4 \\
\hline 16 & 4660 & 37 & 3 & 4 & 2 & 3 & 4 & 0 & 2 & 5.4 \\
\hline 17 & 5100 & 19 & 2 & 2 & 1 & 1 & 3 & 0 & 1 & 5.3 \\
\hline 18 & 5100 & 19 & 2 & 1 & 2 & 0 & 2 & 0 & 1 & 5.3 \\
\hline 19 & 5200 & 19 & 1 & 0 & 0 & 1 & 4 & 0 & 1 & 5.3 \\
\hline 20 & 5200 & 19 & 1 & 1 & 0 & 3 & 2 & 0 & 1 & 5.3 \\
\hline 21 & 5000 & 19 & 1 & 0 & 0 & 2 & 1 & 0 & 0 & 0 \\
\hline 22 & 4660 & 19 & 2 & 0 & 0 & 0 & 2 & 0 & 0 & 0 \\
\hline 23 & 4660 & 19 & 1 & 0 & 0 & 0 & 1 & 0 & 0 & 0 \\
\hline
\end{tabular}

Altitude ranged from near sea level $(215 \mathrm{~m})$ to $5200 \mathrm{~m}$ over the expedition. The 5 Lake Louise Self-Assessment Questionnaire (LLSAQ) categories of headache $(\mathrm{H})$, dizziness $(\mathrm{D})$, gastrointestinal symptoms $(\mathrm{GI})$, fatigue $(\mathrm{F})$, and sleep disturbances $(\mathrm{S})$ are each scored from 0 to 3 of increasing severity. Median LLSAQ scores and number of subjects recording scores of $\geq 3$ for each day are also shown, together with daily incidences. AMS, acute mountain sickness.

decreased again on acclimatization at this altitude and showed no increase on further ascent.

At altitude, compared with control data at $215 \mathrm{~m}$, oxygen saturations decreased significantly (Figure 3), and a strong negative correlation was noted $(\rho=-0.59$, $P=.001)$. Blood pressure also weakly correlated with altitude, with both systolic and diastolic measures increasing with increasing altitude $(\rho=0.18, P=.003$,

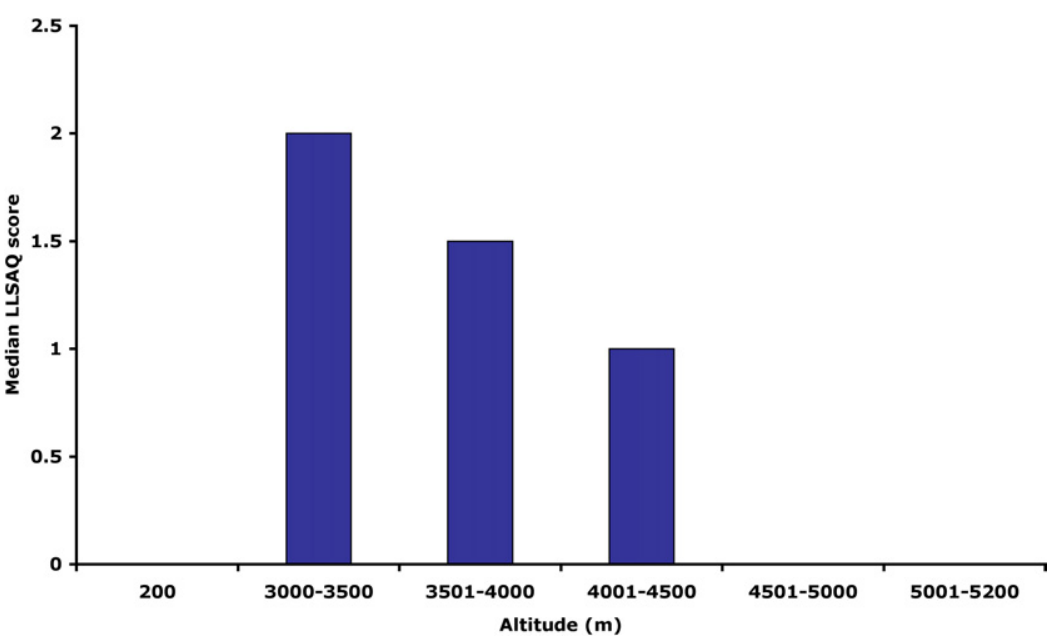

Figure 2. Median Lake Louise Self-Assessment Questionnaire (LLSAQ) scores versus altitude. Different numbers of recordings were collected within the various altitude ranges. The median scores were identified for each altitude range. 


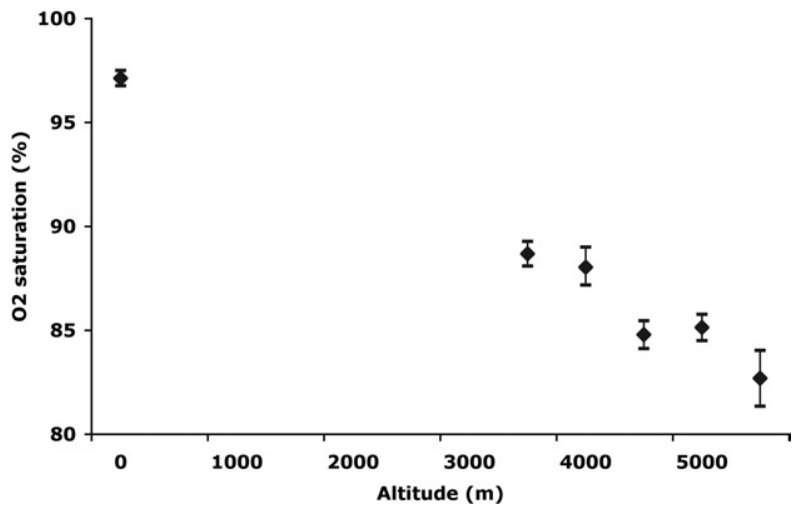

Figure 3. Peripheral oxygen $\left(\mathrm{O}_{2}\right)$ saturation versus altitude. Preexercise oxygen saturation measurements (diamonds) at increasing altitude (mean $\pm 95 \%$ confidence interval) show an association of oxygen saturation with altitude $($ rho $=-0.59, P=.001$ ).

and $\rho=0.36, P=.004$, respectively). The pulse rate for the group increased significantly from a mean of 70 beats/min $( \pm 6.5)$ at sea level to 83 beats/min $( \pm 2.2)$ at $3500 \mathrm{~m}(P=.02$; Figure 4$)$. The raised pulse rates at altitude remained elevated but did not correlate with further altitude gain $(\rho=0.02, P=.6)$.

Pulse rate showed a weak positive correlation with increasing LLSAQ score $(\rho=0.15, P<.001$; Figure $5 \mathrm{a})$, and oxygen saturation, a weak negative correlation $(\rho=-0.16, P=.005$; Figure 5b). No relationship was identified between blood pressure and LLSAQ score ( $\rho=0.00$ and $\rho=0.02$ for systolic and diastolic blood pressure, respectively).

Table 2 lists mean values for all physiological measurements. Subjects recording LLSAQ $\geq 3$ on a particular day showed lower oxygen saturations on that day

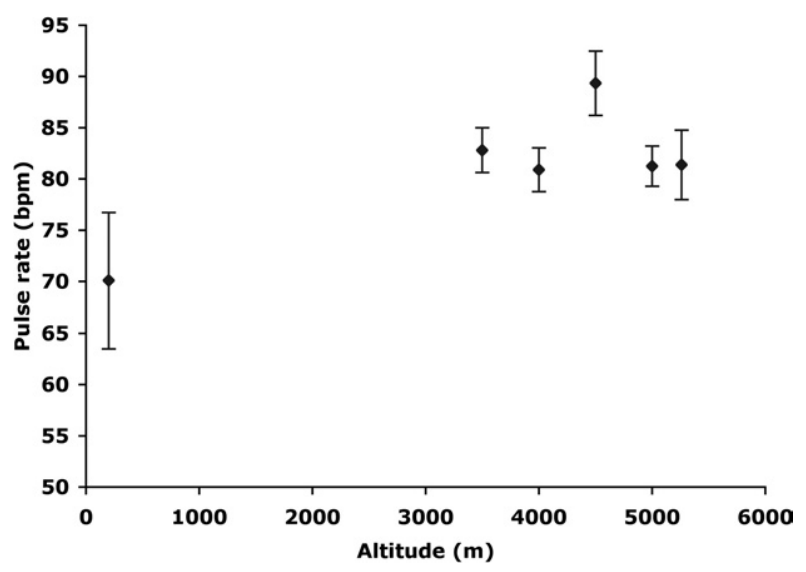

Figure 4. Pre-exercise pulse rate measurements (diamonds) are shown for the whole group at increasing altitude (mean $\pm 95 \%$ confidence interval). A significant increase on ascent to $3500 \mathrm{~m}$ is shown, with no clear association with further altitude gain (rho $=0.02, P=$ .6). bpm, beats per minute.
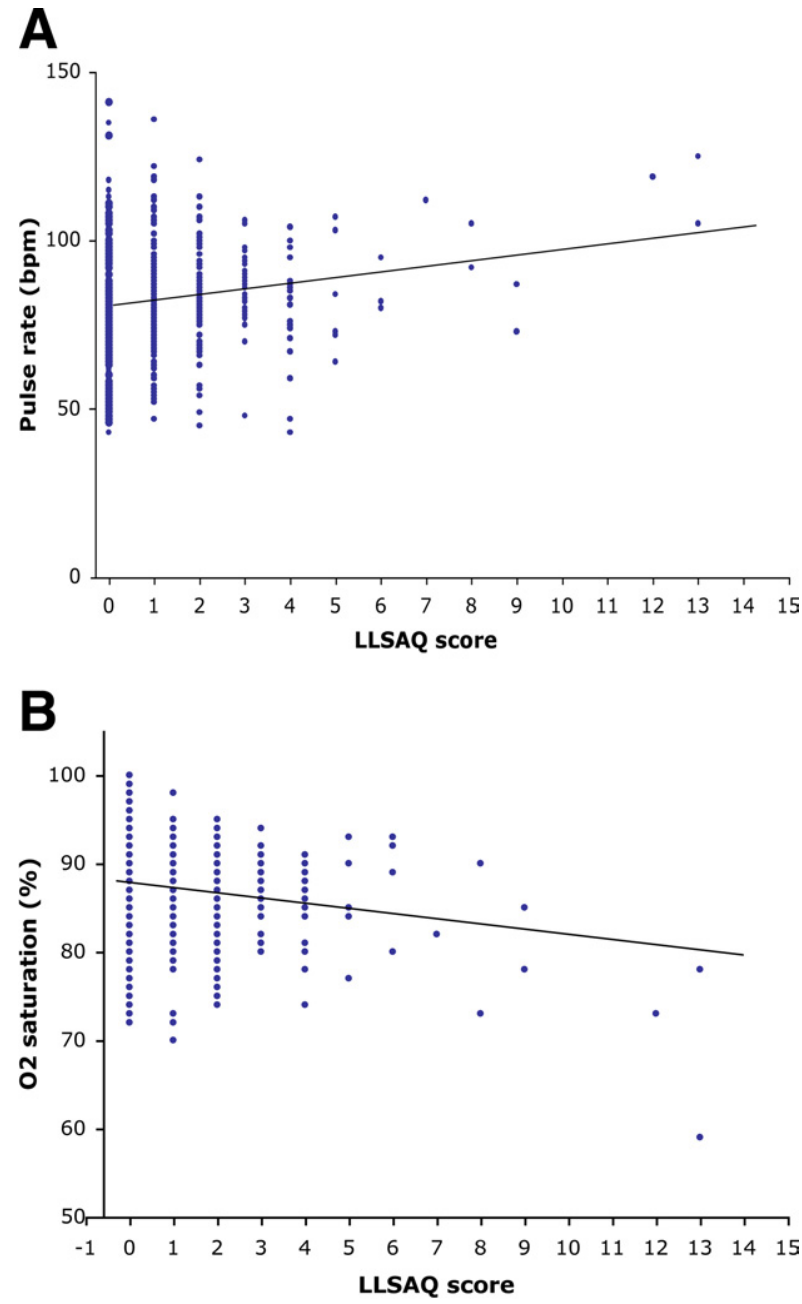

Figure 5. Correlation of Lake Louise Self-Assessment Questionnaire (LLSAQ) against physiological measurements. (A) Association of pulse rate with LLSAQ score (regression line $\mathrm{r}=0.15, P<.001$ ). (B) Association of oxygen $\left(\mathrm{O}_{2}\right)$ saturation with LLSAQ score (regression line $\mathrm{r}=-0.16, P=.005)$. Correlation lines represent Spearman's correlation coefficients. bpm, beats per minute.

compared with subjects who did not have scores indicative of $\operatorname{AMS}(P=.013)$. Mean oxygen saturations for subjects experiencing AMS was $84.9 \%( \pm 1.6)$, and for participants without AMS symptoms, it was $87.1 \%$ $( \pm 0.5)$. Subjects who had severe AMS recorded a mean oxygen saturation of $78.3 \%( \pm 2.3)$ compared with $87.1 \%( \pm 0.5)$ for subjects who did not $(P=.001)$. Lower oxygen saturations were noted in participants recording scores indicative of severe AMS; Figure 6 shows severe AMS scores are overrepresented at lower oxygen saturations.

Exercise at altitude caused significant falls in oxygen saturation from rest. Control data at $215 \mathrm{~m}$ show no significant decrease in oxygen saturation on exertion (Figure 7, red line). Upon exercise at altitude, oxygen 
Table 2. Physiological measurements before and after exercise

\begin{tabular}{|c|c|c|c|c|c|}
\hline Physiological measure & $\begin{array}{c}\text { No AMS } \\
\text { LLSAQ }<3(n=606)\end{array}$ & $\begin{array}{c}A M S \\
L L S A Q \geq 3(n=71)\end{array}$ & $\begin{array}{c}\text { Severe AMS } \\
\text { LLSAQ } \geq 7(n=11)\end{array}$ & $\begin{array}{l}\text { Diff. mean } \\
\text { AMS/no. }\end{array}$ & $\begin{array}{c}\text { Diff. mean } \\
\text { severe AMS/no. }\end{array}$ \\
\hline $\mathrm{O}_{2}$ sats before exercise & $87.1( \pm 0.5)$ & $84.9( \pm 1.3)$ & $78.3( \pm 2.3)$ & $\begin{array}{c}2.2 \\
P=.013\end{array}$ & $\begin{array}{c}8.8 \\
P=.001\end{array}$ \\
\hline $\mathrm{O}_{2}$ sats after exercise & $81.4( \pm 0.4)$ & $78.7( \pm 1.4)$ & $70.1( \pm 4.5)$ & $\begin{array}{c}2.7 \\
P=.001\end{array}$ & $\begin{array}{c}11.3 \\
P=.003\end{array}$ \\
\hline $\mathrm{O}_{2}$ sats after $1-\mathrm{min}$ rest & $86.2( \pm 0.4)$ & $84.4( \pm 1.3)$ & $71.2( \pm 4.4)$ & $\begin{array}{c}1.8 \\
P=.02\end{array}$ & $\begin{array}{c}15 \\
P=.046\end{array}$ \\
\hline Pulse rate before exercise & $82.4( \pm 1.3)$ & $86( \pm 3.4)$ & $102.3( \pm 12.0)$ & 3.6 & $\begin{array}{c}19.9 \\
P=.004\end{array}$ \\
\hline Pulse rate after exercise & $131.5( \pm 1.8)$ & $140.2( \pm 5.4)$ & $150.6( \pm 12.9)$ & $\begin{array}{c}8.7 \\
P=.005\end{array}$ & $\begin{array}{c}19.1 \\
P=.014\end{array}$ \\
\hline Pulse rate after 1-min rest & $85.3( \pm 2.4)$ & $91.1( \pm 4.4)$ & $128.4( \pm 12.8)$ & 7.1 & $\begin{array}{c}43.1 \\
P=.005\end{array}$ \\
\hline Systolic BP before exercise & $135.9( \pm 1.1)$ & $133.6( \pm 2.9)$ & $130.6( \pm 6.2)$ & 2.3 & 5.3 \\
\hline Systolic BP after 1-min rest & $142.5( \pm 1.3)$ & $139.4( \pm 4.5)$ & $144.5( \pm 9.9)$ & 3.1 & 2 \\
\hline Diastolic BP before exercise & $83.1( \pm 0.9)$ & $79.9( \pm 2.4)$ & $78.1( \pm 4.6)$ & 3.2 & 5 \\
\hline Diastolic BP after 1 min rest & $84.3( \pm 0.9)$ & $81.6( \pm 2.7)$ & $77.6( \pm 5.9)$ & 2.7 & 6.7 \\
\hline
\end{tabular}

Physiological data represented as mean $( \pm 95 \% \mathrm{CI})$ before and after exercise for the group with no acute mountain sickness (AMS) and the AMS group. A subgroup of severe AMS is also included. Each daily recording of the Lake Louise Self-Assessment Questionnaire (LLSAQ) is coupled to the physiological measurements for that person on that day (n), giving a direct comparison of symptoms to physiological data. The difference in means between no AMS and AMS/severe AMS are indicated. Statistical significance was judged as $P<.05$. The "n" represents number of data recordings of physiological signs coupled to the relevant LLSAQ score. Diff., difference; sats, saturations; BP, blood pressure.

saturation decreases significantly at all altitude ranges above $3000 \mathrm{~m}$ (Figure 7, post step). Directly after exercise, subjects scoring LLSAQ scores suggestive of AMS recorded lower oxygen saturations $(P=.001)$ than did subjects with no AMS. After exercise, mean oxygen saturations were $78.7 \%$ ( \pm 1.4$)$ for subjects who had AMS and $81.4 \%( \pm 0.4)$ for subjects who did not have AMS. After 1 minute of rest, mean oxygen saturations

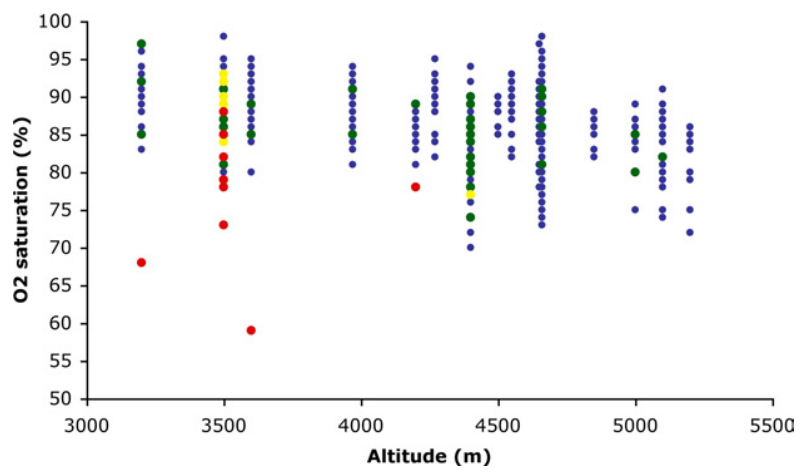

Figure 6. Individual resting pre-exercise peripheral oxygen $\left(\mathrm{O}_{2}\right)$ saturation readings plotted against altitude. Readings are shown corresponding to Lake Louise Self-Assessment Questionnaire (LLSAQ) acute mountain sickness (AMS) scores: $<3$, no AMS (blue dots); 3 to 4, mild AMS (green dots); 5 to 6, moderate AMS (yellow dots); and $\geq 7$, severe AMS (red dots). returned to pre-exercise levels in both AMS subjects and no-AMS subjects.

Participants recording scores indicative of severe AMS (LLSAQ $\geq 7$ ) also showed lower oxygen saturations compared with participants with no AMS after exercise. Mean oxygen saturations after exercise for subjects with severe AMS was $70.1 \%$ ( \pm 4.5$)$, compared

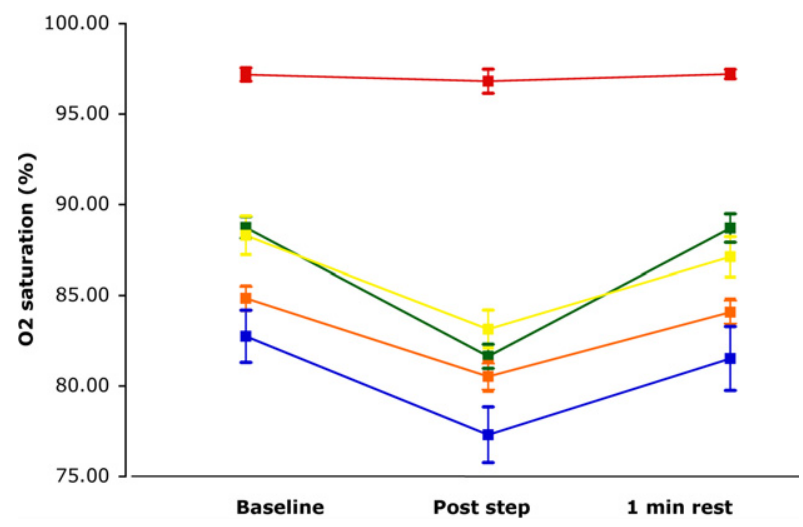

Figure 7. Mean peripheral oxygen $\left(\mathrm{O}_{2}\right)$ saturation results for all recordings at 5 altitude ranges: sea level (red), $3000 \mathrm{~m}$ to $3500 \mathrm{~m}$ (green), $3501 \mathrm{~m}$ to $4499 \mathrm{~m}$ (yellow), $4500 \mathrm{~m}$ to 5000 (orange), and $5001 \mathrm{~m}$ to $5200 \mathrm{~m}$ (blue). Before exercise (Baseline), after 2-minute exercise (Post step), and after 1-minute rest (1 min rest) measurements were averaged separately (mean $\pm 95 \%$ confidence interval). 
with $81.4 \%( \pm 0.4)$ for subjects without AMS symptoms $(P=.003)$. Oxygen saturations recorded by subjects with AMS also remained low after the 1-minute rest period compared with subjects with no severe AMS recorded. For subjects with severe AMS, oxygen saturations remained significantly lower (mean $71.2 \% \pm 4.4$, $P=.02)$ compared with levels before exercise.

Raised pulse rates at rest were noted in participants recording scores indicative of severe AMS. A mean pulse rate of 102.3 beats/min ( \pm 12.0$)$ for subjects who had severe AMS compared with a mean of 82.4 beats/ min $( \pm 1.3)$ for subjects who did not $(P=.004)$. Pulse rate increased with exercise at all altitude ranges, including $215 \mathrm{~m}$. Directly after exercise, subjects with LLSAQ scores suggestive of AMS recorded higher pulse rates than did subjects with no AMS $(P=.005)$. Mean pulse rate for AMS subjects after exercise was 140.2 beats/min ( \pm 5.4 ); for no-AMS subjects, it was 131.5 beats $/ \mathrm{min}$ $( \pm 1.8)$. Pulse rates returned to pre-exercise levels in both AMS subjects and no-AMS subjects after a 1-minute rest. Participants recording scores indicative of severe AMS (LLSAQ $\geq 7$ ) also showed higher pulse rates compared with participants with no AMS after exercise. Post-exercise mean pulse rate recorded for the severe AMS group was 150.6 beats/min ( \pm 12.9$)$, compared with 131.5 beats/min $( \pm 1.8)$ recorded for the no-AMS group $(P=.014)$. The raised pulse rates after exercise were prolonged in subjects recording severe AMS, compared with subjects with no AMS recorded. For the severe AMS group, pulse rates remained significantly higher than pre-exercise levels 1 minute after rest (mean $128.4 \pm 12.8 ; P=.006$ ).

There was no difference in blood pressure between AMS subjects and no-AMS subjects $(P=.371$ and $P=$ .146 for systolic and diastolic, respectively), and there was no difference for subjects who had severe AMS $(P=.296$ and $P=.223$ for systolic and diastolic, respectively). Blood pressure measurements after exercise showed no differences between groups (systolic, $P=.372$; diastolic, $P=.289$ ).

\section{Discussion}

Our study identified an overall AMS incidence of $84.0 \%$ in this group of adolescents. Daily incidences ranged between $0 \%$ and $39 \%$, with greatest numbers experiencing symptoms on initial ascent to $3500 \mathrm{~m}$ and also a further peak on ascent to $4660 \mathrm{~m}$. The LLSAQ scores tended to decrease on further, more gradual ascent, suggesting that the ascent profile was adequately slow to allow acclimatization for the majority of the group. An inverse relationship between increasing altitude and oxygen saturation was confirmed $(P=.001)$, and a rise in both mean pulse rate and blood pressure with ascent was highlighted. Persons experiencing symptoms of AMS tended to record higher pulse rates and lower oxygen saturations, and exercise-induced drops in oxygen saturation and raised pulse rates were found to be more prolonged in subjects who had severe AMS compared with no-AMS subjects $(P=.02$ and $P=.006$, respectively).

Participants displaying symptoms of AMS tended to record higher pulse rates and lower oxygen saturations at rest than did participants with no AMS symptoms. Comparison of means between the 2 sets of data shows a significant difference between the symptomatic AMS group and the group not experiencing symptoms. We have also shown correlations between both oxygen saturation and pulse rate with LLSAQ score. These data give an indication that severity of altitude-related symptoms show a relationship with lower oxygen saturations and higher pulse rates, but the strength of the correlations and differences between group means are not large, and assumptions taken from these data should be treated with caution. The study data show a possible association but by no means confirm it as fact.

Exercise tests at altitude resulted in an increase in mean pulse rates and decrease in mean oxygen saturations. The decrease in mean oxygen saturation was solely observed at altitude, with no identifiable exercise-induced drop in saturations at sea level. That was observed in all groups. Exercise-induced drops in oxygen saturation and raised pulse rates were, however, more prolonged in subjects with severe AMS; they appeared to have taken longer to recover after exercise, with 1 minute of inactivity being inadequate to return measurements to pre-exercise levels.

Our results show a high incidence of AMS compared with some other studies. ${ }^{4}$ The group ascended rapidly to $3500 \mathrm{~m}$, compared with the more gradual ascent profiles of other studies. ${ }^{9}$ The group experienced the greatest altitude-related symptoms after this rapid ascent, which could account for potential exaggeration of AMS severity. The daily incidences over the expedition were, however, similar to those found by Dallimore et al. ${ }^{9}$ and overall closely reflected the prevalence seen in the pilot study (91.7\%). The need for vigilance and monitoring of symptoms was stressed to the group, especially on initial ascent, perhaps leading to overrecording of symptoms. Most participants experienced a headache, even when doing well, and a combination of factors such as fatigue, dehydration, unaccustomed exercise, and a bout of nonaltitude-related respiratory tract infections may all have led to overestimation of AMS symptoms. The majority of persons recording AMS were able to participate fully in the expedition, but some were undoubtedly more than 
mildly affected by altitude. That was reflected in their lack of general well-being, poor appetite, fatigue, low mood, high LLSAQ scores, and to some extent, in their lower oxygen saturations. This finding demonstrates that a number of different approaches to assessing altituderelated illness should be taken into account.

Upon further ascent above $3500 \mathrm{~m}$, the recording of AMS symptoms decreased. That decrease may be due in part to the evacuation of 1 severely affected participant on day 7, thereby reducing the potential for further severe scores recorded. It is also likely that the few days spent at $3500 \mathrm{~m}$ was adequate in the majority of cases to allow acclimatization to occur, and therefore, further gradual ascent did not bring about any great increase in AMS symptoms. This acclimatization trend has been observed previously in studies of both adolescents and adults. ${ }^{5,14}$

\section{STUDY LIMITATIONS}

The study has provided further insight in AMS development in adolescents, although it does have a number of limitations that should be noted. A total of 38 adolescents was included in the study, providing reasonable numbers of recordings. The data obtained give some insight into the likely trends and lines of further investigation. Notably, only 19 subjects recorded data at higher altitudes, above $4700 \mathrm{~m}$, potentially skewing results. Equally, with only 7 persons recording severe AMS, the comparison between groups has inevitable shortcomings. Potential limitations to the baseline data collection include that a single dataset on each person was obtained in humid conditions in New Delhi within 24 hours after a long haul flight.

The study participants were highly motivated and enthusiastic about the project, but it is not possible to confirm the accuracy of recordings taken. The participants were supervised for the first 4 days to ensure consistency, as far as possible. The adolescents then performed their own unsupervised recordings after this period, providing a potential area of inaccuracy in the results. The equipment used for all physiological measurements was identical in make and model but its reliability under expedition conditions has not been confirmed. The accuracy of the pulse oximetry equipment has not been reliably tested at $<75 \%$ saturations, resulting in potentially inaccurate results for subjects with low saturation readings, especially at the higher altitudes reached. ${ }^{13}$ Given that recordings were done early in the morning, temperature may be a factor for both equipment accuracy and the effect on circulation to fingertips. Hence, the decrease in oxygen saturation with altitude may be, to some extent, an artifact of decreasing tem- peratures with altitude and its effect on peripheral circulation. Other equipment factors such as movement artifact and excess light may also be significant, as previously suggested by Luks et al. ${ }^{13}$ Measurements were performed in the mornings outside the tents under direct sunlight. Every attempt was made to keep movement to a minimum during recordings but measurements, especially after exercise, may have a certain amount of potential error. Undoubtedly, by keeping time, place (outside tents), equipment, recording protocols, and exercise regimens constant throughout the expedition, potential errors were kept to a minimum.

Overall, our data show that as altitude and AMS scores increase, oxygen saturations decrease and pulse rates tend to increase. These findings are similar to those of a number of studies recording a correlation between increasing LLSAQ score and AMS and decreased oxygen saturations. ${ }^{15-17}$ The observed drop in peripheral oxygen saturation immediately after exercise was also more marked in participants with AMS symptoms. Recent data suggest that low desaturation after exercise predicts successfully reaching the summit of Aconcagua. ${ }^{18} \mathrm{~A}$ delayed recovery after exercise was observed for participants with severe AMS in this study, and may suggest a reduced ability to compensate for increased oxygen demand with exercise in high altitude environments. Other studies have used recorded decreases in oxygen saturations as a predictive tool for AMS. ${ }^{16}$ It is not clear from the data that routine monitoring would provide any earlier indication of subsequent AMS development. A number of studies have failed to show any correlation between AMS development and oxygen saturation at all. ${ }^{19}$ Our data identify a general trend of lower oxygen saturations in subjects with AMS, but some subjects with no AMS recorded equally low saturations. This unpredictability suggests pulse oximetry may not be a reliable indicator of altitude illness.

\section{Conclusions}

This study has shown that despite no previous experience of either travel to altitude or the scoring system, the adolescents found the scoring system to be quick and easy to learn, use, and interpret. We have found that persons with serious altitude-related illness are easily identified by a combination of regular monitoring of general well-being, picking up a failure to participate in daily activities, and experience of leaders to assess those struggling. The LLSAQ scoring system complimented this approach and undoubtedly aided in raising awareness among the adolescents themselves of persons affected within the group. A combination of sensible monitoring and involvement of groups in the LLSAQ scoring 
system are likely to improve safety of adolescents at altitude. Physiological changes with altitude merit further study as there appears to be a certain amount of association between changes in physiological measurements and altitude-related symptoms. We have not, however, provided any further concrete evidence to suggest that monitoring of vital signs will contribute to safer travel in the mountains. Vigilance combined with appropriate ascent profiles and experienced support are key.

\section{Acknowledgments}

The British Schools Exploring Society (BSES) supported this work. Thanks to all the members of the BSES Ladakh 2008 expedition who diligently recorded their symptoms and physiological measurements. Thanks also go to the University of Bristol for the loan of equipment and to the BSES for practical support.

\section{References}

1. Scrase E, Laverty A, Gavlak JC, et al. The Young Everest Study: effects of hypoxia at high altitude on cardiorespiratory function and general well-being in healthy children. Arch Dis Child. 2009;94:621-626.

2. Imray $\mathrm{C}$, Booth $\mathrm{A}$, Wright $\mathrm{A}$, Bradwell A. Acute altitude illnesses. BMJ. 2011;343:411-417.

3. West J, Schoene R, Milledge J. Ventilatory response to hypoxia and carbon dioxide. In: High Altitude Medicine and Physiology. 2nd ed. London: Hodder Arnold; 2007:51-66.

4. Moraga FA, Osorio JD, Vargas ME. Acute mountain sickness in tourists with children at Lake Chungara (4400 m) in northern Chile. Wilderness Environ Med. 2002;13:31-35.

5. Imray $\mathrm{CH}$, Kennedy $\mathrm{CH}$, Pattinson $\mathrm{K}$, Brearey $\mathrm{S}$, Wright A. Self-assessment of acute mountain sickness in adolescents: a pilot study. Wilderness Environ Med. 2004; 15:202-206.

6. Honigman B, Theis MK, Koziol-McLain J, et al. Acute mountain sickness in a general tourist population at moderate altitudes. Ann Intern Med. 1993;118:587-592.

7. Bloch J, Duplain H, Rimoldi SF, et al. Prevalence and time course of acute mountain sickness in older children and adolescents after rapid ascent to 3450 meters. Pediatrics. 2009;123:1-5.
8. Yaron M, Waldman N, Niermeyer S, Nicholas R, Honigman B. The diagnosis of acute mountain sickness in preverbal children. Arch Pediatr Adolesc Med. 1998;152: 683-687.

9. Dallimore J, Rowbotham EC. Incidence of acute mountain sickness in adolescents. Wilderness Environ Med. 2009;20: 221-224.

10. Sutton JR, Coates G, Houston CS, Hackett PH, Oelz O. The Lake Louise consensus on the definition and quantification of altitude illness. In: Sutton JR, Coates G, Houston CS, eds. Hypoxia and Mountain Medicine. Burlington, VT: Queen City Printers; 1992:327-330.

11. Roach RC, Bartsch P, Oelz O, Hackett PH. The Lake Louise AMS Scoring Consensus Committee. The Lake Louise acute mountain sickness scoring system. In: Sutton JR, Houston CS, Coates G, eds. Hypoxia and Molecular Medicine. Burlington, VT: Queen City Printers, 1993: 272-274.

12. Levett DZ, Martin DS, Wilson MH, et al. Design and conduct of Caudwell Xtreme Everest: an observational cohort study of variation in human adaptation to progressive environmental hypoxia. BMC Med Res Methodol. 2010;10:98.

13. Luks AM, Swenson ER. Pulse oximetry at high altitude. High Alt Med Biol. 2011;12:109-119.

14. Purkayastha SS, Ray US, Arora BS, et al. Acclimatization at high altitude in gradual and acute induction. $J$ Appl Physiol. 1995;79:487-492.

15. Moraga FA, Pedreros CP, Rodriguez CE. Acute mountain sickness in children and their parents after rapid ascent to 3500 m (Putre, Chile). Wilderness Environ Med. 2008;19: 287-292.

16. Karinen HM, Peltonen JE, Kahonen M, Tikkanen HO. Prediction of acute mountain sickness by monitoring arterial oxygen saturation during ascent. High Alt Med Biol. 2010;11:325-332.

17. Roach RC, Greene ER, Schoene RB, Hackett PH. Arterial oxygen saturation for prediction of acute mountain sickness. Aviat Space Environ Med. 1998;69:1182-1185.

18. Lazio MP, Van Roo JD, Pesce C, Malik S, Courtney DM. Postexercise peripheral oxygen saturation after completion of the 6-minute walk test predicts successfully reaching the summit of Aconcagua. Wilderness Environ Med. 2010;21: 309-317.

19. O'Connor T, Dubowitz G, Bickler PE. Pulse oximetry in the diagnosis of acute mountain sickness. High Alt Med Biol. 2004;5:341-348. 